Document downloaded from:

http://hdl.handle.net/10251/98706

This paper must be cited as:

J. Antonino-Daviu; Pons Llinares, J.; Lee, SB. (2016). Advanced Rotor Fault Diagnosis for Medium-Voltage Induction Motors Via Continuous Transforms. IEEE Transactions on Industry Applications. 52(5):4503-4509. doi:10.1109/TIA.2016.2582720

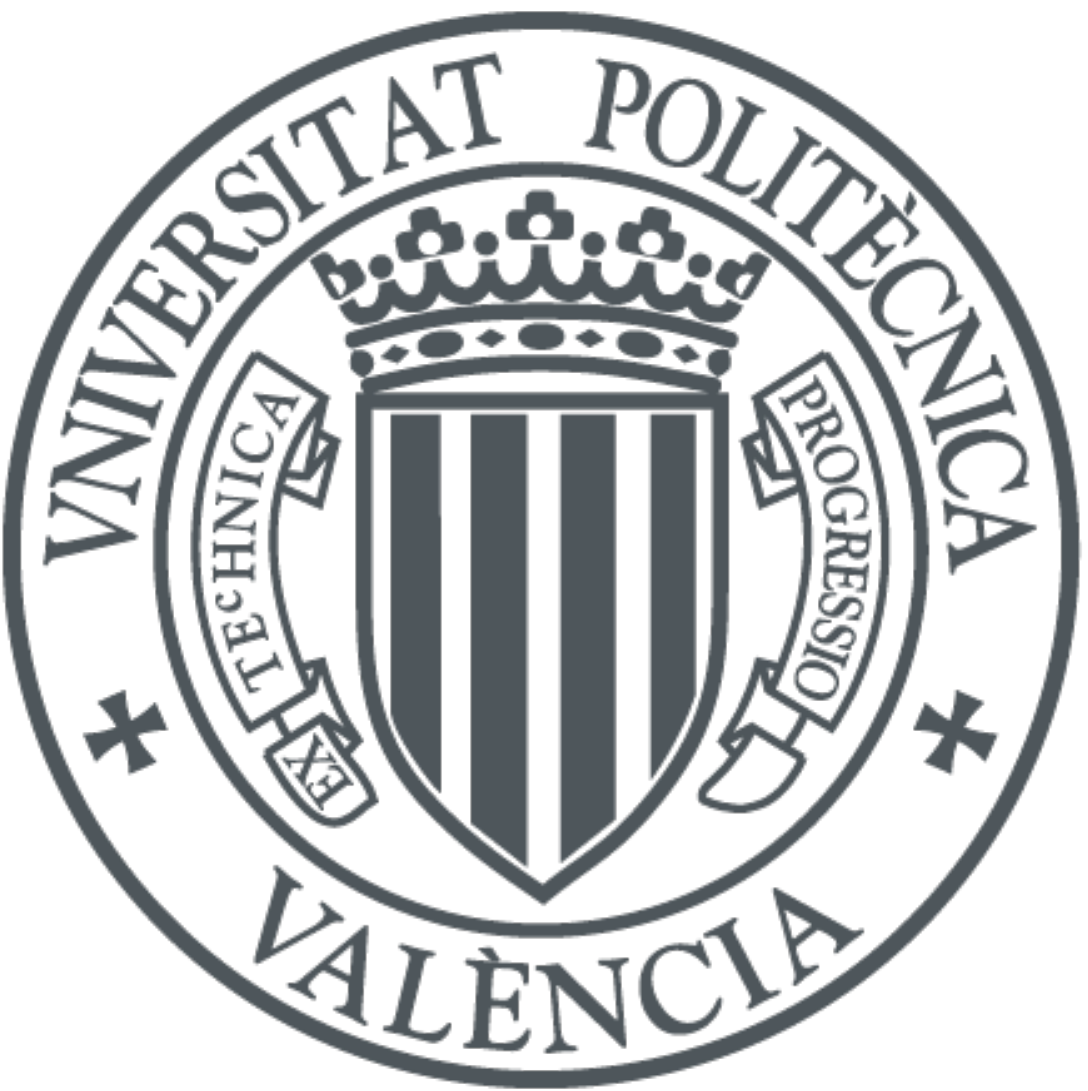

The final publication is available at

http://doi.org/10.1109/TIA.2016.2582720

Copyright Institute of Electrical and Electronics Engineers

Additional Information 


\title{
ADVANCED ROTOR FAULT DIAGNOSIS FOR MEDIUM VOLTAGE INDUCTION MOTORS VIA CONTINUOUS TRANSFORMS
}

\author{
Copyright Material PCIC Europe
}

Paper No. PCIC-LO-77

\author{
Jose A. Antonino-Daviu \\ Senior Member IEEE \\ Universitat Politècnica València \\ Institute for Energy Engineering \\ Valencia \\ SPAIN
}

\author{
Joan Pons-Llinares \\ Member IEEE \\ Universitat Politècnica València \\ Institute for Energy Engineering \\ Valencia \\ SPAIN
}

\author{
Sang Bin Lee \\ Senior Member IEEE \\ Korea University \\ Dept. Electrical Engineering \\ Seoul \\ KOREA
}

\begin{abstract}
A number of field case studies for rotor fault diagnosis on medium voltage induction motors operating in a petrochemical plant are presented in this paper. The methodology employed is based on analyzing the induction motor startup current with advanced signal processing tools (Continuous Transforms) that enable a capture of a 'complete picture' of the rotor condition. Indeed, unlike the classical tools that often rely on the detection of few fault frequencies, these new tools allow extraction of the evolution of a wide range of fault components during the startup transient and steady-state evolutions, which enables improved reliability. This is crucial in medium-high voltage motors, where a false diagnosis may result in significant expense due to inspection, repair, or forced outage. An additional contribution of the work is its immunity to external voltage supply disturbances, which introduce components that are not related to the failure, and which are difficult to detect with classical tools. The results of the work prove how the advanced continuous tools enable an improved visualization of the fault components, distinguishing them from the other components that are not linked to the failure.
\end{abstract}

Index Terms - Fault diagnosis, induction motors, spectral analysis, transient analysis, wavelet transforms.

\section{INTRODUCTION}

An incorrect assessment of the rotor condition in a large induction motor can have severe economic consequences, as well as other losses in less tangible terms [1]. If the rotor is incorrectly diagnosed as faulty (false positive), there will be significant costs derived from the unnecessary motor disassembly, transportation and inspection at the motor repair shop (leaving aside the loss of production revenue). In addition, the credibility of the maintenance staff may be seriously compromised. On the other hand, if the motor is incorrectly diagnosed as healthy (false negative), the fault can worsen and an unexpected forced outage of the motor and driven process may take place. This may lead to even more severe repercussions (economic and material costs caused by unplanned production shutdowns, user safety hazard, and damage to the company's reputation). In spite of all these consequences, the likelihood of an incorrect diagnosis of the rotor condition is not negligible at all, at least when employing the techniques that are nowadays widespread in industry.

The most extensively used method in industry for induction motor rotor condition assessment, known as Motor Current Signature Analysis (MCSA), is based on the analysis of the stator current measured during motor operation by applying the Fast Fourier Transform (FFT) [2-5]. The idea is to evaluate the amplitudes of the frequency components linked with the corresponding failure. In the case of rotor damage, there are two families of frequencies amplified by the fault (see expressions (1) and (2), where $s=s l i p, f=$ supply frequency, $k=$ integer in (1) and $k / p=1,3,5 \ldots$ in (2)). The most relevant components are known as sideband harmonics and are obtained from (1) for $k=1$. The sidebands (lower and upper) appear around the fundamental frequency, as illustrated in Fig 1.

$$
\begin{gathered}
f_{b b 1}=[1 \pm 2 k s] f \\
f_{b b 2}=\left[\frac{k}{p}(1-s) \pm s\right] \cdot f
\end{gathered}
$$

Evaluation of the amplitude of the lower sideband component, relative to that of the fundamental component, can be used to determine the severity of the rotor failure. Typically, sideband amplitudes between $\sim 55 \mathrm{~dB}$ and $\sim 45 \mathrm{~dB}$ are indicative of incipient rotor damage. In case of having values in such range, proper actions should be adopted by the maintenance staff. Note that in most of the cases, damages can be present in the rotor cage for prolonged time intervals (even years) without substantially affecting the machine operation. However, unscheduled outage of the motor and process due to secondary damage caused by protrusion of damaged rotor cage fragments is not uncommon. An example of a $3.3 \mathrm{kV}, 450$ $\mathrm{kW}$ induction motor driving a gasoline transfer pump at an oil refinery is shown in Fig. 2. The motor tripped during operation due to stator winding insulation damage caused by contact between a broken rotor bar and stator endwinding, as can be seen in Figs. 2(a)-(b). Such motor failures not only can cause a forced outage, but also increase the safety risks in the petrochemical industry.

In spite of the fact that the conventional MCSA has led

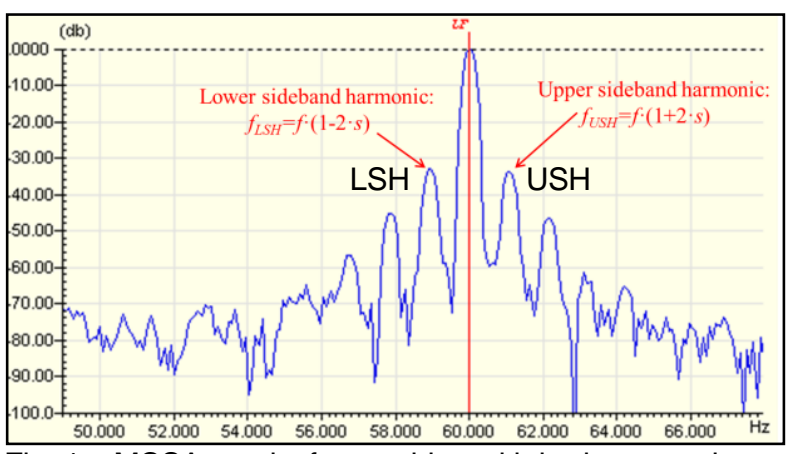

Fig. 1 MCSA results for machine with broken rotor bars 
to correct results in many applications, some cases have been reported in the literature in which the application of MCSA may lead to erroneous diagnostics (either false positives- diagnosing a healthy machine as faulty, or false negatives- diagnosing a faulty machine as healthy) [6-14]. The most common cases leading to false positives or false negatives, when applying the MCSA approach, are summarized in Table I.

\section{DISCRETE AND CONTINUOUS ANALYSIS TOOLS}

Due to the drawbacks of the MCSA approach, a significant effort has been employed to develop alternative approaches that can overcome the incorrect diagnosis provided by the conventional method. One of these attempts consists of extending the analysis to the currents demanded by the machine during startup transient operation. This approach, recently named as Advanced Transient Current Signature Analysis (ATCSA), relies on the application of advanced signal processing tools to track the time-frequency evolutions of fault components. More specifically, unlike the classical method that is based on identifying the single characteristic frequency components in the FFT spectrum for evaluating their amplitude, the underlying idea of the new approach is to study how certain frequency components evolve over time $[10,14,16]$. Fault-related components have very specific time-frequency evolutions that are unlikely to be caused by other phenomena. Hence, the detection of these evolutions, if present, is a very reliable indicator of the existence of the fault.

Analysis of the stator startup transient has recently drawn much attention due to the potential advantages over MSCA $[10,14,16]$. The variation of slip during the startup regime (that occurs across a wider slip range in line started machines) leads to particular evolutions of fault-related components. The application of proper signal

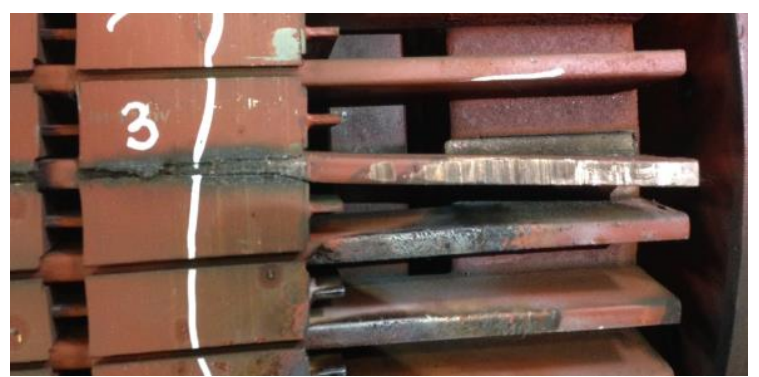

(a)

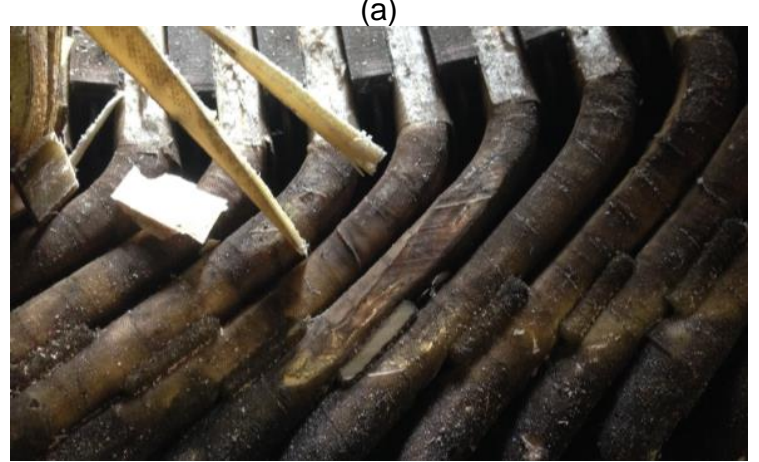

(b)

Fig. 2 Forced outage of 3.3 kV, 450 kW gasoline transfer pump induction motor due to rotor bar damage; (a) rotor bar detachment from end ring and damage in rotor core due to arcing; (b) damage in stator endwinding due to protrusion of rotor bar processing tools enables_detection of such evolutions, confirming the presence of the fault. It is even possible to determine the fault severity with proper indicators, as will be commented later. The application of the transientbased approach requires minimum startup duration longer than 0.5 seconds, which is easily obtained in most industrial motors rated above $3.3 \mathrm{kV}$.

Advanced Transient Current Signature Analysis (ATCSA) requires the application of signal processing tools that are able to provide a time-frequency representation of the analyzed signal (e.g. startup current) to enable visualization of the time evolution of fault-related frequencies. These tools are known as Time-Frequency Decomposition (TFD) tools. There is a wide variety of TFD tools [17]. In the context of the considered application, they can be classified into two main groups:

1) Discrete transforms: these are usually simpler tools, with relatively low computational burden. Some of them can be easily applied since they are available in commercial software packages. They also enable the easy introduction of fault severity indicators based on their resulting signals. The main problem is that the interpretation of the resulting signals is less clear compared to that of the continuous transforms, with regards to the extraction of fault-related evolutions. They are usually less flexible and they enable the simultaneous visualization of a lower number of components' evolutions than their continuous counterparts [18].

An example of these transforms is the Discrete Wavelet Transform (DWT). Fig. 3 shows the results of applying the DWT to the startup current for a machine with one broken rotor bar [10], where $s$ is the measured startup current, and $a_{8}, d_{8}, d_{7}, d_{6}, d_{5}$ represent signals associated with a certain frequency range, as shown in the figure. The tool enables detection of the evolutions of some rotor fault related harmonics (in this case the lower and upper sideband harmonics) through the oscillations appearing in the signals resulting from the analysis. The difference between Fig. 3(a) and (b) is clear, where the patterns created by the evolutions of the fault harmonics can be observed in Fig. 3(b). Moreover, since the amplitudes

TABLE I

COMMON CAUSES LEADING TO FALSE POSITIVES /NEGATIVES WHEN APPLYING MCSA

\begin{tabular}{|c|c|c|}
\hline \multirow{2}{*}{$\begin{array}{c}\text { Actual } \\
\text { machine } \\
\text { condition }\end{array}$} & \multicolumn{2}{|c|}{ Result of MCSA diagnostics } \\
\hline & HEALTHY & FAULTY \\
\hline HEALTHY & TRUE NEGATIVE & $\begin{array}{l}\text { FALSE POSITIVE } \\
\text { - Low frequency load } \\
\text { torque oscillations [2- } \\
4,10] \\
\text { - Interference from } \\
\text { rotor axial cooling } \\
\text { ducts }[1,3,5,13] \\
\text { - Rotor core magnetic } \\
\text { anisotropy [15] }\end{array}$ \\
\hline FAULTY & $\begin{array}{l}\text { FALSE NEGATIVE } \\
\text { - Non-adjacent bar } \\
\text { breakages }[7-8,11] \\
\text { - Outer bar } \\
\text { breakages in double } \\
\text { cage rotors }[6,12] \\
\text { - Erroneous speed } \\
\text { estimation } \\
\text { - Diagnosis under } \\
\text { light- or no-load } \\
\text { conditions [10] }\end{array}$ & TRUE POSITIVE \\
\hline
\end{tabular}


(and, hence, the energy) of the wavelet signals are higher for severe faults, quantification indicators based on the energy of these signals can be developed. For this particular case, a quantification indicator could be, for instance, based on the energy of $d_{7}$ since a substantial portion of the lower sideband harmonic, LSH, evolves in that signal). A possible indicator, proposed in previous works is given by (3) and relates the energy of the signal $d_{7}$ (denominator of the quotient in (3)) to the total startup current signal energy (numerator) during the considered interval. In equation (3), $i_{j}$ is the value of the th sample of the current signal; $d+(J)$ is the $j$ th element of the detail signal $d_{7} ; N_{s}$ is the number of samples of the signal in the startup transient, and $N_{b}$ is the number of samples between the origin of the signals and the extinction of the oscillations due to border effect $[10,14]$.

For medium-high voltage motors, a value of this indicator lower than $50 \mathrm{~dB}$ is indicative of the existence of rotor damage.

$$
\gamma_{D E}(d B)=10 \cdot \log \left[\frac{\sum_{j=N b}^{N s} i_{j}^{2}}{\sum_{j=N b}^{N s}\left[d_{7}(j)\right]^{2}}\right]
$$

When applying the DWT, several parameters such as mother wavelet or number of decomposition levels must be selected by the user. Experience shows that mother wavelets such as dmeyer and high-order Daubechies have provided very satisfactory results. The detailed guidelines for selecting these parameters can be found in [14], [19].

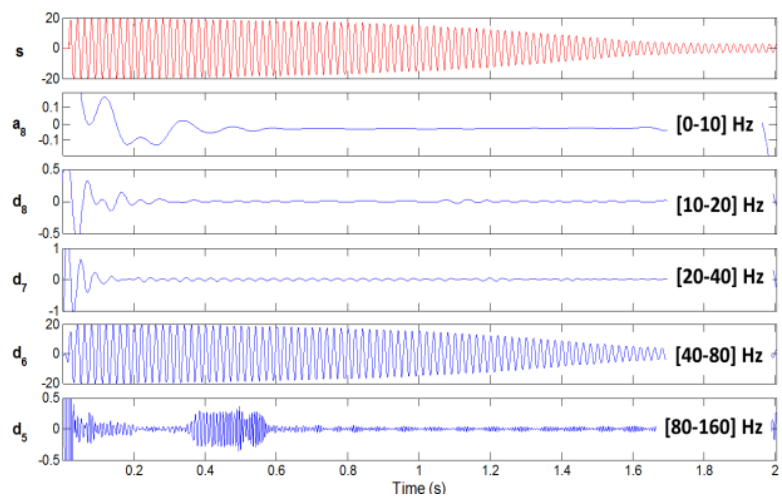

(a)

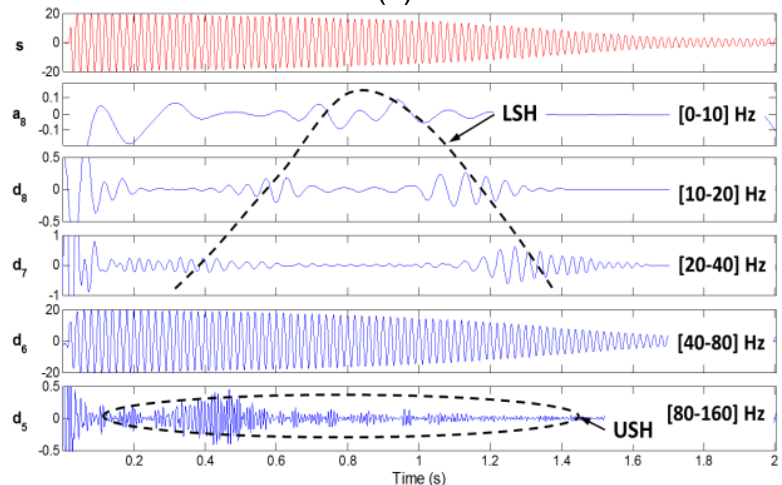

(b)

Fig. 3 Discrete Wavelet Transform (DWT) of the startup current for: (a) healthy machine, (b) machine with 1 broken bar
2) Continuous transforms: Unlike their discrete counterparts, continuous transforms provide a continuous 'picture' that resembles an 'X-ray photograph' of the analyzed signal that shows its full time-frequency content, as shown in Fig. 4. In this time-frequency map, the y-axis represents the range of frequencies analyzed, and the $x$ axis represents the time interval of the signal. The color at any point of the map at coordinates $x 0, y 0$ is related to the amplitude of the frequency component $y 0$ at the specific time $x 0$. An intense color in the map indicates that that frequency component has a strong presence in the analyzed signal at that specific time instant.

There are a number of variants of continuous transforms, depending on the approach to obtain the distribution of the signal energy in the time vs frequency (t-f) map (energy $t-f$ density). In this regard, Short Time Fourier Transform (STFT), Continuous Wavelet Transform (CWT), Wigner-Ville Distribution (WVD), or Choi-Williams Distributions (CWD) among others, have been successfully applied in the literature. Technical details of these transforms can be found in [17-18].

The application of these continuous transforms is often more complex but they provide a more complete representation of the time-frequency content of the analyzed signal [18]. This enables tracking of the evolution of a higher number of fault components evolutions with high resolution. Logically, the higher the

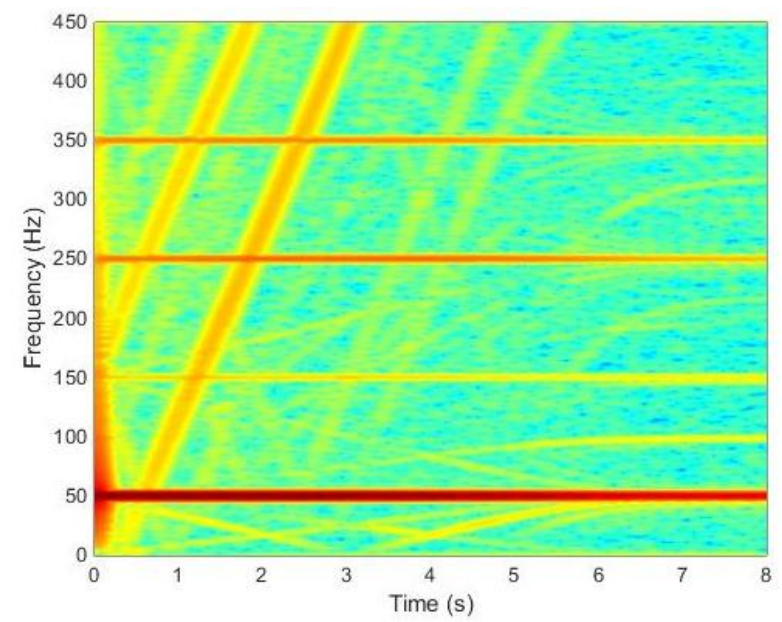

(a)

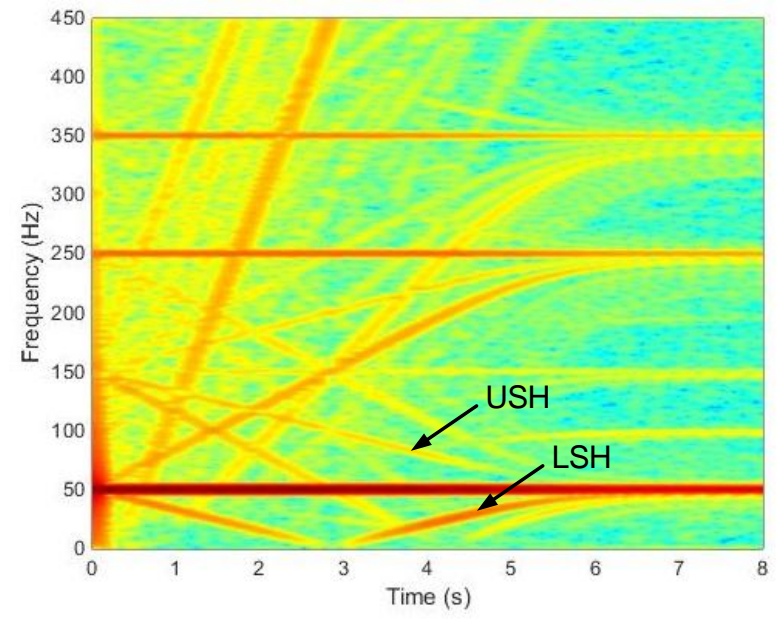

(b)

Fig. 4 Short Time Fourier Transform (STFT) of the startup current for: (a) healthy machine, (b) machine with 1 broken bar 
number of tracked evolutions, the more reliable the diagnostic becomes. The main criticism of the continuous transforms is the computational burden that their application requires. The computational requirements significantly change depending on many aspects (range of analyzed frequencies, frequency resolution, duration of the analyzed signal, etc...). Due to this fact, these tools are more suitable for off-line analysis.

The STFT analysis of the startup current for a healthy machine (Fig. 4(a)) and for a machine with 1 broken bar (Fig. 4(b)) [18] is shown in Fig. 4. Note the large number of fault harmonics that can be tracked in the second case. In Fig. 4(b), the LSH and upper sideband harmonic, USH, evolutions have been highlighted.

The aim of this paper is to present actual examples of the application of continuous transforms to the startup current of medium voltage motors operating in petrochemical plants. In the examples, the advantages of each diagnostic approach (MCSA and ATCSA using discrete and continuous tools) are evaluated. Although the case studies presented in this paper are for direct-online started motors, the methodology is applicable for the cases of soft-started and even inverter-fed motors, as shown in previous publications [20]-[21].

\section{RESULTS: APPLICATION OF CONTINUOUS TRANSFORMS TO MEDIUM VOLTAGE MOTORS}

\section{A. Diagnosed motors}

Three medium voltage pump motors operating in an oil refinery have been analyzed in this paper. The first motor, denoted M1, is a $6.6 \mathrm{kV}, 270 \mathrm{~kW}$ pump motor that was diagnosed with a broken rotor bar. Disassembled inspection of the motor confirmed that it had a broken bar, and it was repaired. The startup current of the motor before and after motor repair was obtained and analyzed for evaluation of the proposed method. The second motor, $\mathrm{M} 2$, is a $6.6 \mathrm{kV}, 800 \mathrm{~kW}$ motor influenced by an external interference that introduces components that are not related to the failure. These components that appear intermittently can cause confusion when diagnosing the motor with MCSA or discrete transforms. The third motor, $\mathrm{M} 3$, is a $3.3 \mathrm{kV}, 200 \mathrm{~kW}, 2$ pole crude oil pump motor, for which MCSA produced a false rotor fault indication due to rotor core magnetic anisotropy, as described in [15]. The startup data of this motor was analyzed to observe if startup analysis can provide reliable diagnosis. The ratings of the three motor samples $\mathrm{M1}, \mathrm{M} 2$, and $\mathrm{M} 3$ are summarized in Table II.

\section{B. Results and discussion}

The results of MCSA applied for the case of motor M1 before (Fig. 5(a)) and after (Fig. 5(b)) rotor cage repair are shown in Fig. 5. In the former case, the MCSA result reveals a significant amplitude of the $\mathrm{LSH}(-44.7 \mathrm{~dB})$ that is indicative of rotor failure. However, the chance of the signature being a false positive case must be ruled out. Hence, to ratify the diagnostic, the ATCSA was applied,

TABLE II

RATED CHARACTERISTICS FOR THE DIAGNOSED MOTORS

\begin{tabular}{ccccc}
\hline Motor & $\begin{array}{c}\text { Power } \\
(\mathrm{kW})\end{array}$ & $\begin{array}{c}\text { Voltage } \\
(\mathrm{V})\end{array}$ & Poles & Frequency $(\mathrm{Hz})$ \\
\hline M1 & 270 & 6600 & 4 & 60 \\
M2 & 800 & 6600 & 6 & 60 \\
M3 & 200 & 3300 & 2 & 60 \\
\hline
\end{tabular}

using both discrete and continuous transforms. The application of the Discrete Wavelet Transform (DWT) to the motor startup current before repair is shown in Fig. 6(a). The wavelet signals reveal clear oscillations that are arranged in concordance with the pattern caused by the evolution of the LSH. Moreover, the fault indicator defined by (3) was computed to have a value of $39.2 \mathrm{~dB}$ was

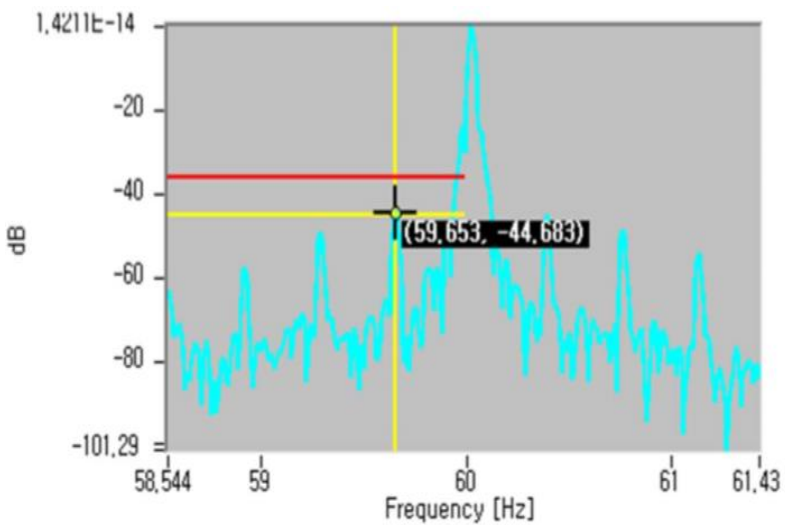

(a)

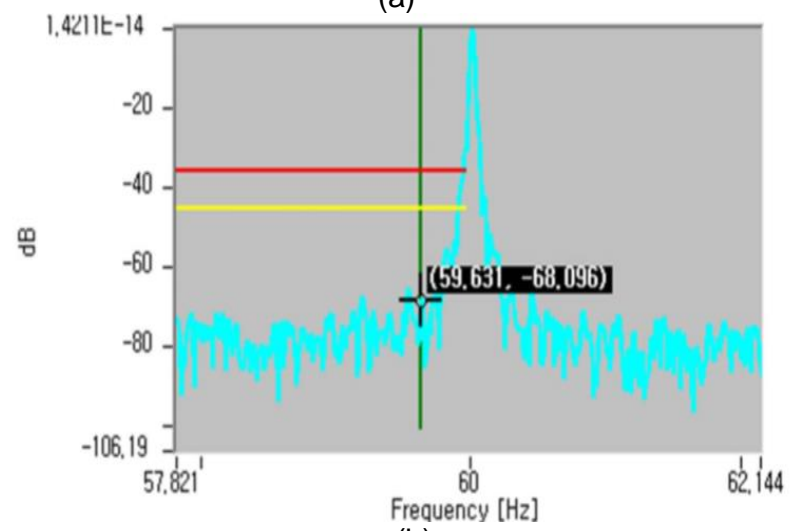

(b)

Fig. 5 MCSA application for motor M1: (a) before repair, (b) after repair

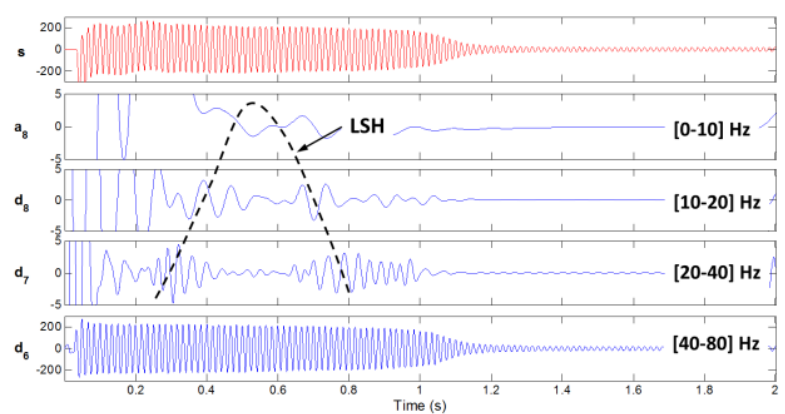

(a)

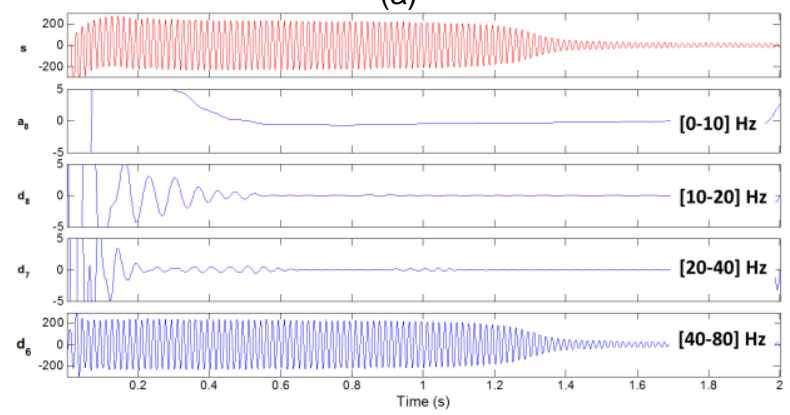

(b)

Fig. 6 DWT application for the motor M1: (a) before repair, (b) after repair. 
obtained. This is indicative of a clear level of rotor failure since it is smaller than the $50 \mathrm{~dB}$ fault threshold. Application of a continuous tool (STFT) results in the same conclusion (Fig. 7(a)) in that a clear V-shaped pattern caused by the LSH evolution can be observed during the startup. This confirms the presence of the rotor fault with a high reliability, avoiding possible false positive indications of the classical tools.

The same analyses were repeated after repairing the motor. Fig. 5(b) reveals the low amplitude of the LSH after repair $(-68 \mathrm{~dB})$, that is indicative of healthy condition. The patterns given by Figs. $6(\mathrm{~b})$ and 7 (b) also confirm this healthy condition of the rotor. Fig. 6 (b) shows very weak pattern of the rotor fault wavelet signals and the value of the indicator $\gamma_{D E}$ was increased to $63.5 \mathrm{~dB}$, which is larger than the $50 \mathrm{~dB}$ threshold. Similarly, no significant LSH traces were found in Fig. 7(b).

MCSA of motor M2 led to the spectrum depicted in Fig. 8. The presence of many components with significant amplitudes located near the $20 \mathrm{~Hz}$ and $40 \mathrm{~Hz}$ and the fundamental frequency were observed, where the source of the interference could not be identified. Diagnosis based on that spectrum is not easy, especially if the speed at which the motor runs is unknown. ATCSA was applied to this case to evaluate its reliability in determining the rotor condition. In the DWT analysis of the startup current, shown in Fig. 9, the LSH pattern is not present, but some oscillations are detected in signal $d_{7}$. They are due to the interference of the unexpected component at $40 \mathrm{~Hz}$. The presence of these components leads to a

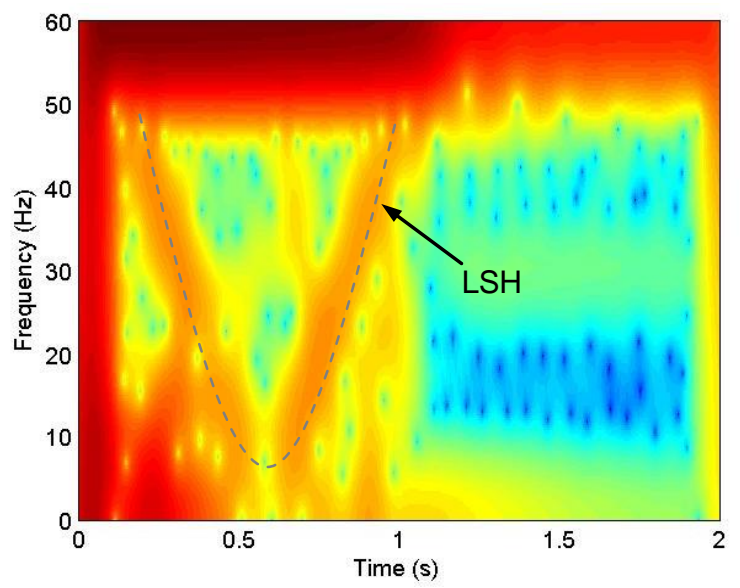

(a)

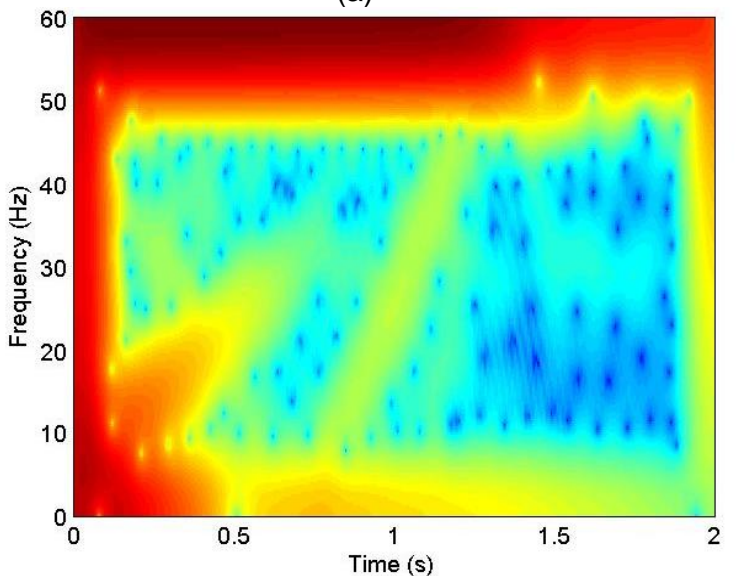

(b)

Fig. 7 STFT application for the motor M1: (a) before repair, (b) after repair. value of the indicator $\gamma_{D E}$ close to $50 \mathrm{~dB}$, which can potentially lead to a false indication in the diagnostics.

The application of the STFT shown in Fig. 10 confirms the presence of two constant components at $20 \mathrm{~Hz}$ and $40 \mathrm{~Hz}$ that are not related to the failure since the $\mathrm{LSH}$ pattern is totally absent. Hence, continuous transforms are very useful for ruling out the chance of false fault indication, providing immunity against the external disturbance that introduces components at multiples of 20 $\mathrm{Hz}$.

The MCSA measurements of the rotor fault sidebands, $f_{b b 1}$, obtained from M3 are shown in Fig. 11. The motor was pulled and inspected since the fault component increased over time to $-49.36 \mathrm{~dB}$, as shown in Fig. 11 . Visual inspection and a series of off-line tests showed that the rotor was in good condition, and the investigation presented in [15] revealed that the false alarm is produced by rotor core magnetic anisotropy. The 20 and

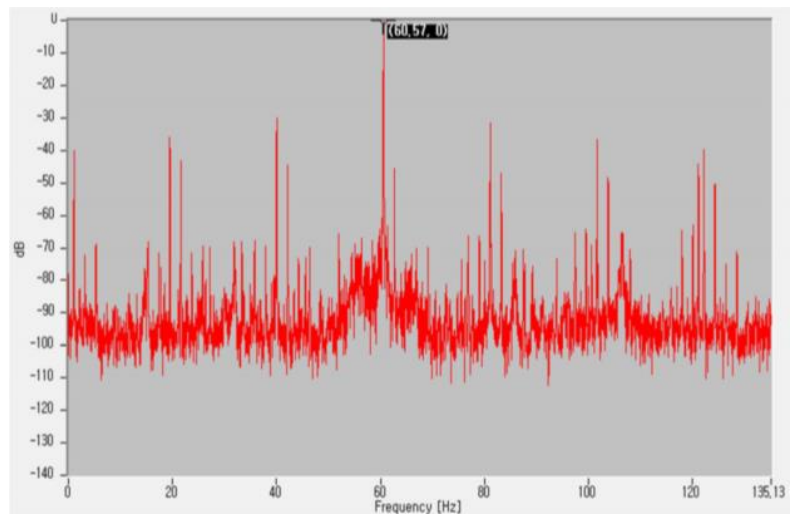

Fig. 8 MCSA application for motor M2.

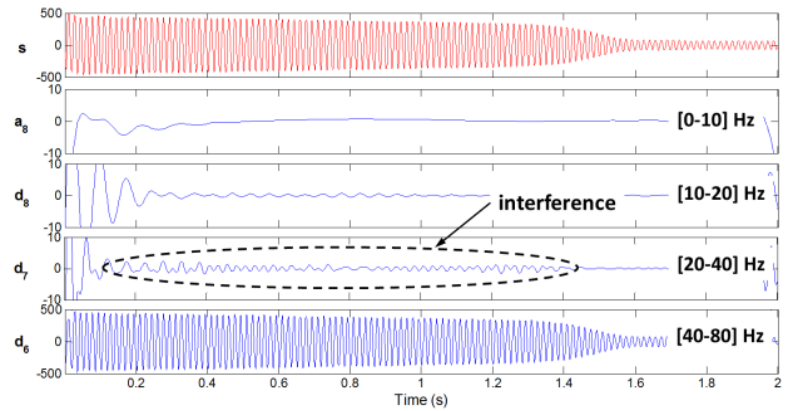

Fig. 9 DWT application for the motor M2.

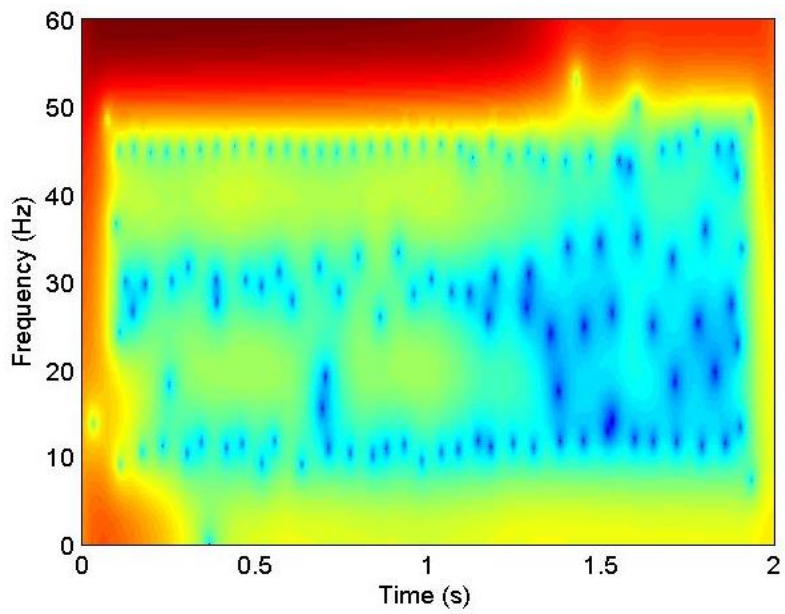

Fig. 10 STFT application for the motor M2. 
$40 \mathrm{~Hz}$ external interference similar to that shown in Fig. 8 was also observed for the case of M3. ATCSA analysis was performed on this motor to verify if the DWT and STFT startup analysis methods can reliably diagnose the motor as "healthy".

Although a clear LSH pattern is not present in the DWT analysis of the startup current shown in Fig. 12, noticeable oscillations were observed in $d_{7}$ and $d_{8}$ due to the interference at 20 and $40 \mathrm{~Hz}$. The oscillations make it difficult for the user to make a judgment on rotor condition with high confidence. The value $\gamma_{D E}$ was calculated as ???, which is ??? $50 \mathrm{~dB}$, which can lead to a false rotor fault indication. The results of the STFT shown in Fig. 13 confirm that the oscillations observed with DWT were caused by the $20 \mathrm{~Hz}$ and $40 \mathrm{~Hz}$ components not related to the failure. A weak LSH pattern produced by the inherent asymmetry in the rotor, as in the case of M1 with a healthy rotor (Fig. 6(b)) can be observed in Fig. 13. The results show that the rotor is in good condition since the LSH pattern in Fig, 13 is not strong enough, as in the case of Fig. 6(a), to suspect a broken bar. The reason the startup test method is not influenced by the magnetic anisotropy is because the flux is distributed on the surface of the rotor at startup due to eddy current rejection of the rotor cage [15]. With the flux concentrated at the rotor surface, it is not influenced by the anisotropy of the core. Fig. 13 clearly shows that the potential false alarm produced by magnetic anisotropy and external interference with MCSA or DWT can be avoided with STFT.

\section{CONCLUSIONS}

Several field studies concerning the application of continuous transforms to diagnose the rotor condition of medium voltage motors operating in petrochemical plants were presented in this paper. The results show that analysis of the motor startup current provides reliable detection of rotor faults. It was also shown that continuous transforms lead to more reliable diagnostic conclusions compared to discrete transforms since continuous transforms enable a better and clearer visualization of fault components' evolutions, as well as tracking a larger number of fault harmonics. These characteristics allow the proposed method to provide immunity against false alarms since components not related to the failure can be clearly separated from the fault-related ones. This is not always possible when using the conventional MCSA or discrete transforms for rotor fault detection.

\section{REFERENCES}

[1] C. Yang, T-J. Kang, D. Hyun, S. Lee, J. AntoninoDaviu, J. Pons-Llinares, "Reliable Detection of Induction Motor Rotor Faults Under the Rotor Axial Air Duct Influence," IEEE Trans. on Ind. Appl., vol. 50, no. 4, pp 2493-2502, July/Aug 2014.

[2] W.T. Thomson, M. Fenger, "Current signature analysis to detect induction motor faults," IEEE Ind. Appl. Mag., July/Aug. 2001, pp. 26-34.

[3] W.T. Thomson, "On-line current monitoring - the influence of mechanical loads or a unique rotor design on the diagnosis of broken rotor bars in induction motors," Proc. of ICEM, pp. 1236-1240, 1992.

[4] H. Kim, S. Lee, S. Park, S. Kia, G. Capolino, "Reliable detection of rotor faults under the

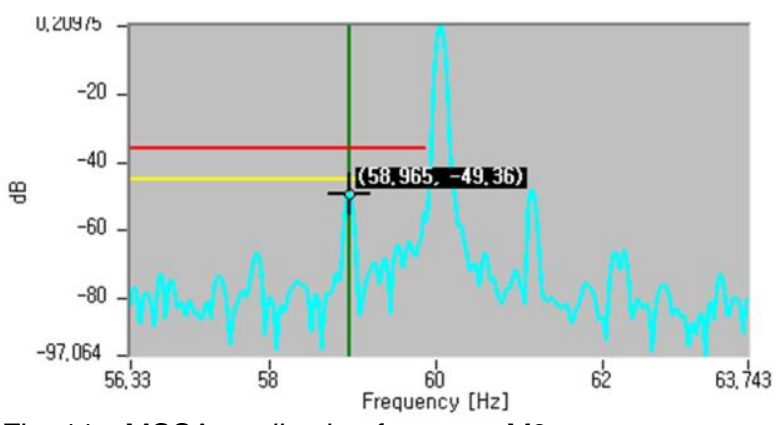

Fig. 11 MCSA application for motor M3.

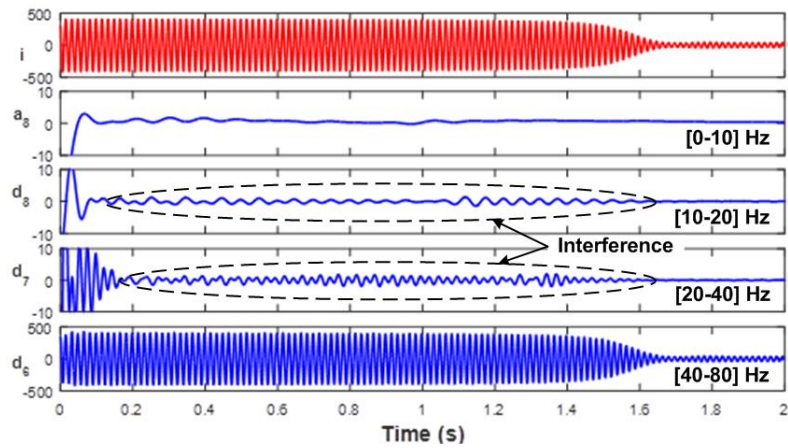

Fig. 12 DWT application for the motor M3.

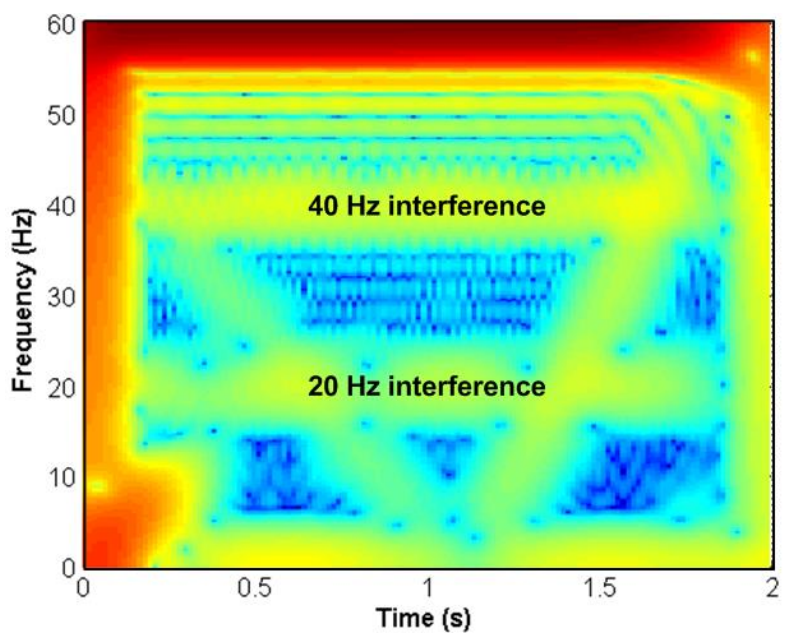

Fig. 13 STFT application for the motor M3.

influence of low frequency load torque oscillations for applications with speed reduction couplings," Proc. of IEEE SDEMPED, pp.56-63, Sept. 2015.

[5] A. Bellini, et al., "On-field experience with on-line diagnosis of large induction motors cage failures using MCSA," IEEE Trans. on Ind. Appl., pp. 10451053, vol. 38, no. 4, July/Aug. 2002.

[6] S. Williamson, and M.A.S. Abdel-Magied, "Steady state analysis of double cage induction motors with rotor cage faults," IEE Proc. - Elec. Pwr. Appl., vol. 134, no. 4, pp. 199-206, July 1987.

[7] G.Y. Sizov, A. Sayed-Ahmed, C. Yeh, N.A.O. Demerdash, "Analysis and diagnostics of adjacent and nonadjacent broken-rotor-bar faults in squirrelcage induction machines," IEEE Trans. on Ind. Elec., vol. 56, no. 11, pp. 4627-4641, Nov. 2009.

[8] T.J. Sobczyk, and W. Maciolek, "Diagnostics of rotor-cage faults supported by effects due to higher MMF harmonics," Proc. of Power Tech. Conf., vol. 2, pp. 5-9, June 2003. 
[9] J. Antonino-Daviu, V. Climente-Alarcon, J. PonsLlinares, E. Wiedenbrug, "Advanced Rotor Assessment of Motors Operating under Variable Load Conditions in Mining Facilities," Proc. of IEEE ECCE, 2014.

[10] J. A. Antonino-Daviu, M. Riera-Guasp, J. R. Folch, and M. Pilar Molina Palomares, "Validation of a new method for the diagnosis of rotor bar failures via wavelet transform in industrial induction machines," IEEE Trans. on Ind. Appl., vol. 42, pp. 990-996, 2006.

[11] M. Riera-Guasp, M. Fernandez-Cabanas, J. A. Antonino-Daviu, M. Pineda-Sanchez, C.H.R. Garcia, "Influence of Nonconsecutive Bar Breakages in Motor Current Signature Analysis for the Diagnosis of Rotor Faults in Induction Motors," IEEE Trans. on Ener. Convers., vol. 25, no.1, pp. 80-89, 2010.

[12] J. Park, B. Kim, J. Yang, K. Lee, S.B. Lee, E.J. Wiedenbrug, M. Teska, and S. Han, "Evaluation of the Detectability of Broken Rotor Bars for Double Squirrel Cage Rotor Induction Motors," Proc. Of IEEE ECCE, 2010.

[13] S. Lee, J. Hong, S.B. Lee, E.J. Wiedenbrug, M. Teska, H. Kim, "Evaluation of the Influence of Rotor Axial Air Ducts on Condition Monitoring of Induction Motors," IEEE Trans. on Ind. Appl., vol.49, no.5, pp.2024,2033, Sept.-Oct. 2013.

[14] M. Riera-Guasp, J. A. Antonino-Daviu, M. PinedaSanchez, R. Puche-Panadero, and J. Perez-Cruz, "A General Approach for the Transient Detection of Slip-Dependent Fault Components Based on the Discrete Wavelet Transform," IEEE Trans. Ind. Electron., vol. 55, pp. 4167-4180, 2008.

[15] S. Shin, J. Kim. S.B. Lee, C. Lim; E.J. Wiedenbrug, "Evaluation of the Influence of Rotor Magnetic Anisotropy on Condition Monitoring of Two-Pole Induction Motors," IEEE Trans. on in Ind. Appl., vol. 51, no. 4, pp. 2896-2904, Jul./Aug. 2015.

[16] R. Burnett, J.F. Watson, S. Elder, "The detection and location of rotor faults within three phase induction motors" Conference Record of ICEM, 1994.

[17] J. Pons-Llinares, V. Climente-Alarcón, F. VedreñoSantos, J. Antonino-Daviu, and M. Riera-Guasp, "Electric Machines Diagnosis Techniques via Transient Current Analysis," Proc. of IECON, 2012.

[18] J. Pons-Llinares, J. Antonino-Daviu, M. RieraGuasp, S.B. Lee, T. Kang, C. Yang, "Advanced Induction Motor Rotor Fault Diagnosis via Continuous and Discrete Time-Frequency Tools," IEEE Trans. on Ind. Elec., vol.62, no.3, pp.17911802, March. 2015.

[19] J. Antonino-Daviu, M. Riera-Guasp, J. Roger-Folch, F. Martínez-Giménez, A. Peris, "Application and Optimization of the Discrete Wavelet Transform for the Detection of Broken Rotor Bars in Induction Machines". Applied and Computational Harmonic Analysis, Elsevier, vol. 21, pp. 268-279, September 2006.

[20] J. Corral Hernandez, J. A. Antonino-Daviu, V. Climente-Alarcon, J. Pons-Llinares, V. FrancesGaliana, "Transient-based rotor cage assessment in induction motors operating with soft-starters," IEEE
Transactions on Industry Applications, vol. 51, no.5, pp. 3734-3742, Sep/Oct. 2015.

[21] J. Pons-Llinares, D.Moriñigo-Sotelo, O. DuquePerez, J. Antonino-Daviu and M. Perez-Alonso, "Transient Detection of Close Components though the Chirplet Transform: Rotor Faults in Inverter-fed Motors," Proc. of the IEEE IECON 2014, Dallas, USA, 2014

\section{VITA}

Jose A. Antonino-Daviu received his MS and PhD degrees in Electrical Engineering, both from the Universitat Politècnica de València, in 2000 and 2006, respectively. He also received his BS in Business Administration from Universitat de Valencia in 2012. He worked for IBM during 2 years, being involved in several international projects. Currently, he is Associate Professor in the Department of Electrical Engineering of the mentioned University, where he develops his docent and research work. He has been invited professor in Helsinki University of Technology (Finland) in 2005 and 2007, Michigan State University (USA) in 2010 and Korea University (Korea) in 2014. He is IEEE Senior Member since 2012 and he has published over 100 contributions, including international journals, conferences and books.

Joan Pons-Llinares received the MS degree in Industrial Engineering and the $\mathrm{PhD}$ degree in Electrical Engineering from the Universitat Politècnica de València (UPV, Spain), in 2007 and 2013, respectively. He is currently an Assistant Professor in the Electric Engineering Department of the UPV. His research interests include time-frequency transforms, condition monitoring and diagnostics of electrical machines.

Sang Bin Lee received the BS and MS degrees from Korea University, Seoul, Korea, in 1995 and 1997, respectively, and the $\mathrm{PhD}$ degree from the Georgia Institute of Technology, Atlanta, GA, USA, in 2001, all in Electrical Engineering. From 2001 to 2004, he was with General Electric Global Research Center (GRC), Schenectady, NY, USA. From 2010 to 2011, he was a Research Scientist at the Austrian Institute of Technology, Vienna, Austria. Since 2004, he has been a Professor of electrical engineering at Korea University. His current research interests include protection, monitoring and diagnostics, and analysis of electric machines and drives.

He serves as a Distinguished Lecturer for the IEEE Industry Applications Society (IAS), and an Associate Editor for the IEEE TRANSACTIONS ON INDUSTRY APPLICATIONS for the IEEE IAS Electric Machines Committee (EMC). Dr. Lee was the recipient of twelve prize paper awards from the IEEE IAS, IEEE Power Engineering Society, the IEEE IAS EMC, the Pulp and Paper Industry Committee of IEEE IAS, and the Technical Committee on Diagnostics of the IEEE Power Electronics Society. 